\title{
Distributed Monitoring for Wireless Sensor Networks: a Multi-Agent Approach
}

\author{
Bechar Rachid \\ University of Oran Es-senia, Department of Computer Science, Oran, 31000, Algeria \\ E-mail: rachid_bec@yahoo.fr \\ Haffaf Hafid \\ University of Oran Es-senia, Department of Computer Science, Oran, 31000, Algeria \\ E-mail: haffaf.hafid@univ-oran.dz
}

\begin{abstract}
In this paper, we will present a state of the art in using multi-agent technology for wireless sensor networks where the main goal is the conception of software architecture and the network organization dedicated to optimization, performance analysis, and monitoring. Then we explore in particular, the issues of topology control and works in this field and the use of multi-agent systems. Finally we will propose an agent based algorithm for fault tolerance and topology control in a wireless sensor network. Our proposal consists to embed an agent at each node that is responsible for selecting its parent node or the next hop to the sink when transferring packets. The process of parent change is based on the computation of a fault tolerance degree which is calculated periodically by the agent in cooperation with neighboring nodes, in addition to other parameters such as the number of hops, the energy and the quality of link. Simulation results show that this method of changing parent allows a fault tolerant network with enhanced life time compared with the collect tree protocol CTP used for data gathering in a wireless sensor network.
\end{abstract}

Index Terms-Wireless sensor networks, multi-agent systems, monitoring, topology.

\section{INTRODUCTION}

The ad hoc nature of wireless sensor networks (WSN) [1], its cost which is continuously decreasing and the easy deployment are among factors that have contributed to increasing applications exploiting this technology.

Research in this domain is active as WSNs also pose problems implicating several disciplines to solve them. In addition to the usual problems studied in computer networks (wired and wireless networks) generally interested in routing, localization, transfer, control, surveillance and security, WSNs have the problem of energy consumption to be optimized in order to have reliable and fault tolerant networks with a reduction of energy consumption and a certain quality of service.

The multi-agent systems (MAS) [2] have a principle that can be easily adapted and integrated in complex systems due to their fully decentralized approach. They are specially used to model phenomena where global behavior emerges from the local behavior of system entities and/or components. These components have the ability to percept, process, act and react in their environment.

The complexity of wireless sensor networks structure and the distributed processing capacity, when the number of nodes increases, conduct us thinking to exploit developments made in multi-agent systems field to improve networks performances and simplify their design.

Indeed, recent trends toward this integration of multiagent approaches in WSN technology are introduced in many levels and operating aspects. So we find works that propose software architectures for applications and services [3, 4, 5, 6, 7]. Others are interested in network organization and cooperation between nodes $[1,8,9,10]$ that clustering has a dominant preoccupation for this category. A lot of works based on agents treat routing problems in WSN with different applications such as in [11] and [12]. Finally, monitoring and mobility are also subject of several studies [13, 14, 15, 8, 16] and [17] where the use of bio-inspired principles seem interesting with multi-agent systems to solve problems related to mobility as well as scheduling tasks.

Also, these works can be divided into two classes: the first considers the sensor network as a multi agent system; it means that application of agent technology consists to implant the same agent for all nodes to have a cooperative functioning between nodes. The second class provides multi-agent systems adapted to the logical structure of a WSN. For example, in a clustered network, we found node agents, cluster agents, and region agents etc ... or agents are adapted to the functional structure in a single node, for example an agent for communication, another for control, another one for coordination,... etc.

Supervision or monitoring is a set of techniques used to increase the performance of WSN, optimize life time and ensure fault tolerance property using all network parameters that prevent or detect dysfunctions in the system.

The physical limits of the wireless node and variation in the wireless links quality are challenges to give high availability of services, in particularly for WSN applications, where it is necessary to create mechanisms 
to identify and reduce or solve the problem of energy hole. The energy hole can be caused by congestion or frequent use of path which is the reason of the premature death of nodes.

The main approach for load balancing and fault tolerance in sensor networks is the maintenance of a topology that guarantees good conditions of transfer. Indeed, the use of multiple paths helps to balance the energy consumption of nodes and divide the flow of packets on all possible paths. This mechanism also reduces the delay and packet loss which is called also transfer with quality of service. The topology used plays an important role in achieving these goals.

Topology control consists in use of network parameters to generate or maintain a topology which warranty a good operating and a better life time to the network. We find in the literature several works on the topology control in wireless sensor networks but a few numbers of studies use agent technology in this field. For this, we propose in this paper a multi-agent approach for topology control in wireless sensor networks to optimize the life time, and improve fault tolerance in the network.

Our work is to use several network parameters of the node and its neighborhood to give a metric called tolerance degree for each node. This metric is calculated by the node itself in cooperation with its neighbors. When transferring packets, this degree will be used to choose qualified nodes to this transfer in order to ensure a better life time with fault tolerance and without affecting the quality of service.

The rest of this paper contains in the following section a classification of works that use multi-agent systems in wireless sensor networks, then a proposal in the same context on the agent based topology control, and finally we will study the performance of this proposal.

\section{USING MAS IN WSN}

The multi-agent approach aims to use multi-agent system in a WSN, each agent is associated to a node and has a proper code that can be executed locally with the ability to move between them for processing or gathering information. The authors of [18] provide the motivation for using this approach. According to [19], it has many advantages:

- Not affected by the network scalability.

- Extensibility and adaptation tasks.

- Energy Efficiency.

- Curative and progressive system.

In recent years, several researches are interested in the WSN distributed processing based on agent technology. Non-exhaustive classification of these contributions is given below according to their objectives and levels of integration in a WSN. Another classification is found in [18] too.

\section{A. Software architecture of applications and services}

These are works with a high level of abstraction that aim to develop architecture which is based on agent technology and adapted to the WSN characteristics.

In [3], S.Mekid (2006) introduced the concept of intelligent sensor in the general case, he proposes architecture for such sensor and explains the necessary features for its operation when it is integrated into a multi-agent environment and the way in which these agents are combined.

Pratik K.Biswas and al. (2008) presented in [4] an interoperable multi agent architecture through layers. The authors demonstrate the effectiveness of their method by comparing the client/server approach and the multi-agent systems in terms of execution time and energy consumption. The work is an extension of an existing model called Interoperable agent model [20]: the new MAS model that includes eight agent types: Sensor Agent (SA), agent management system (AMS), Directory Facilitator (DF), which helps other agents to cooperation, agent communication channel (ACC), Controller synthesizer (CS), Data Manager (DM), Application Agent (AA ) and Agent Interface (AI). These agents are distributed in a sensor network that may contain both wired and IP nodes. The communication between agents is provided by XML messages.

After their work on the MWAC model (2006) (MultiWireless-Agent Communication) and DIAMOND method [5] which is interested in embedded multi-agent systems, especially in wireless infrastructure, the authors presented in [17] a discussion on embedded systems design specifics that use multi-agent systems.

In [6], Smarsly Kay and al. (2011) proposed a system design-based on migrant agents to define a dynamic operation in a WSN according to nodes requirements; this system was really tested on a platform for thermal variations treatment in experimental environment where performances are improved.

The paper [7] proposes a formal model based on realtime temporal logic for multi-agent system specification and evaluation when it is integrated into a WSN environment. Reactive decisional agents are used to take advantage of their ability to cooperation, reaction to events, communication and concurrence.

\section{B. Organization of the network, clustering and cooperation}

The authors of [1] consider WSN as an hierarchical MAS where the highest level is an interface agent that accepts user requests and distributes the tasks to the next lower level agents, which are defined according to geographical criteria and other factors, these agents are managed by regional agents, each region is divided into sub-regions called clusters managed by cluster agents, at the end of this hierarchy were the query agents which are the sensor nodes themselves.

In order to facilitate the design and implementation of 
WSN, Xue Wang and al. (2007) proposed in [8] a model as combination between mobile agents and MAS. The proposed architecture is hierarchically structured according to the roles played by each sensor in the network. The MAS is used for collaboration and mobile agents for data exchange. This model is used for localization and classification of acoustic targets.

In [9], the optimization of energy consumption and reply time is based on MAS applied to a data collection algorithm for emergent events monitoring where the WSN is divided into dynamic clusters, this is defined by the event importance which determines the size and the lifetime of a cluster. Mobile agents traverse the network through cluster heads and itinerary planning is determined by the residual energy and the packet loss degree in the path. Simulation results show that the multiagent model has better performance in terms of energy consumption and replay time.

Logical clustering model adapted to multi-agent operation is presented in [10]. It divides the nodes into a four levels hierarchy: Atom which is the node itself, micro that represents a group of nodes managed by a cluster head, meso which is the upper level clustering grouping a number of clusters of the same area and finally a virtual cluster representing the entire network. This logical structure can change after an event. At each level is assigned a type of agent cooperating with the higher level agent to accomplish the network operation.

\section{Routing}

The problem of routing in WSN was the subject of several works. The paper [21] of A. M. Popescu and al. (2012) presents a survey on different routing approaches especially those based on position. We give here some other works on routing and which use multi agent systems for there implementation.

Min Liu and al. (2012) treated the routing problem in [11]. The paper proposes a new agent based routing algorithm with quality of service in WSN. The MAS is used to manage the topology changes and the flow of communication by participating in routing and maintenance of paths. The method is based on a Swarm Intelligence principle (inspired from the collective intelligence system of insects and other animals). Factors of service quality are the latency, packet loss, energy conservation, etc. The MAS has two agent types: Forward agent FA (to establish a connection with a neighbor in path search) and Reverse agent RA (in response to build a path).

As [11], the authors of [12] proposed in 2012 a model called MAM (Markovian Agent Model) which is based on Swarm intelligence, but using a Markov model. The agent operations are based on local transitions of a node and the influence by the probable transition of the other nodes (local transitions and induced transitions).

\section{Monitoring and Mobility}

The paper [13] of Matthew D. Coles and al. (2009) focused on mobile WSNs and offers a Bayesian model named BNGRAZ (Bayesian network algorithm grazing) for managing mobility. It is bio-inspired model that emulates the behavior of herbivores grazing pastures. The WSN in question contains some mobile nodes to adjust coverage and connectivity. The choice of itinerary taken by a mobile node is based on the probability of disconnection or inaccessibility, this probability is calculated using information provided by neighbors.

As [13] Saamaja Vupputuri and al. (2010) proposed in [14] a similar principle but with the aim of optimizing the life time and satisfy requested quality of service using data collectors that form clusters by changing position, the movements are made according to objective and not by probability.

A self-adaptation strategy for scheduling of tasks in a WSN is presented in [15] and based on the MAS, in addition a mathematical model which is proposed for dynamic tasks allocation. The algorithm has a collective intelligence functioning called PSO (Particle Swarm Optimization algorithm). According to this article, the advantages of applying agent theories and technologies to the WSN are as follows:

- Contribute to formally analyze and design network activities and organization.

- Expand the WSN intelligence and create an autonomous network system.

- Reduce the redundancy of sensing data and data flow.

- Save the energy of the WSN and effectively extend the network lifetime.

- Achieve the dynamic application of the WSN and highly flexible strategy of task scheduling.

Xue Wang and al. (2007) resumed their model of [8] in [16] to implement a trading and tasks sharing system in multimedia WSNs in order to optimize resource management and the classification result. In [5], each node plays a specific role in its neighborhood, this role is determined by an embedded agent in the node itself by cooperation with neighboring nodes agent. If the node is in the area of intersection of multiple clusters, it has the role of liaison or gateway else it has the simple role of data capture, and finally a representative or cluster head is elected in the cluster to manage the set. Mobility and node failures are well handled by this structure.

To optimize the problem of tasks scheduling and data transmission in video WSN, the work presented by Huang Hai-ping and al. (2009) in [17] introduce a set of intelligent algorithms associated to agents based on genetic algorithms, ant colony algorithms or mixed algorithms (Fig.1).

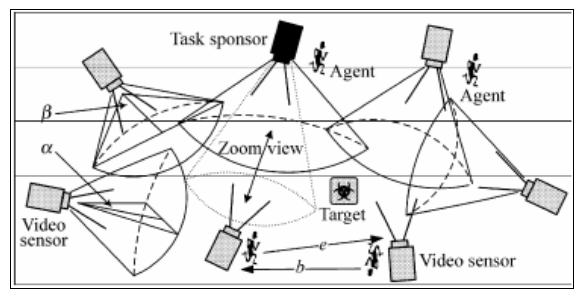

Fig.1. MAS for tasks allocation in [17] 
Each agent (representing a node) decides to participate or not in a target detection regarding to the following criteria:

- S: denotes the status set $\{$ busy, free $\}$ of the current node;

- E: depicts whether or not the current node has enough energy to accomplish the assignment;

- $\alpha$ : angle of vision of the camera on the $\mathrm{x}$-axis (right to left);

- $\beta$ : angle of vision on the y-axis (up and down);

- q: determines the required quality of picture by the monitoring process.

The results show that the algorithms used less energy compared to the performance of AODV protocol.

\section{E. Topology control}

Topology control consists to use multiple network parameters. These parameters can be: radio range, state or role of node.

The majority of works that use multi-agent systems to the topology control are based on hierarchical structures with clusters which is more adapted for MAS running on several levels like [6] and [9]. Other factors may be used in a cooperative manner to obtain a good topology control. This is what we will discuss later in this paper. First, we recall the principles and techniques used in this field and works that exists. According to [22], it exist three main techniques for topology control:

\section{E.1. Power Adjustment Approach}

The power adjustment approach allows nodes to vary their transmission power to reduce energy incurred in transmission. Rather than transmitting at maximum transmission power, nodes work in a collaborative manner to adjust and find the appropriate transmission power to form a connected network. For example in Fig.2, the links N1 - N4 and N2 - N4 are unused by reducing the radio range of these nodes.

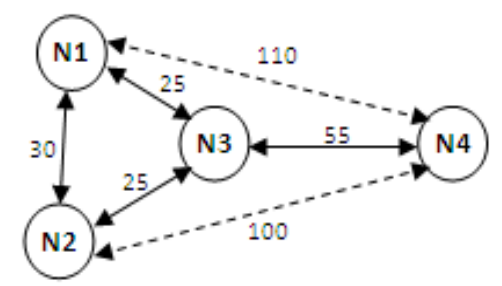

Fig.2. Topology control by adjusting the radio range

Protocols representing this technique are Minimum Energy Communication Network (MECN) [23], and its extension SMECN [24] for Small MECN and COMPOW [25] that conserves energy nodes using the minimum power level that guarantees connectivity of the network.

\section{E.2. Power Mode Approach}

In addition to the techniques used by the MAC layer protocols and when the number of deployed nodes is sufficient, redundancy of nodes can be exploited to get a better topology by changing the state of a node between active and sleep to avoid unnecessary exchanges between nodes. GAF (Geographical Adaptive Fidelity) [26] is an example of a protocol that uses a principle of equivalent nodes, it allows a node to switch between sleep, discovery and active states as shown in Fig.3.

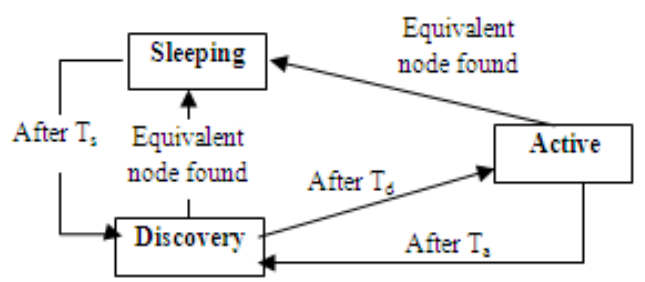

Fig.3. State Transitions in GAF

Also in STEM (Sparse Topology and Energy Management) [27], a maximum of nodes is set to the sleep state for energy network economy. This approach is adapted for applications with low data transfer. Finally, ASCENT (Adaptive Self-Configuring Sensor Network Topologies) [28] is a self-reconfigurable algorithm that allows nodes to locally measure the operating conditions. Based on these conditions, nodes then decide whether they need to participate in routing or not. This algorithm allows a node to choose one state from test, passive, active or sleep.

\section{E.3. Hierarchical structures}

This technique is to find a structure with hierarchical cluster for the network. Choosing cluster heads presents a problem for this technique. Several contributions have tried to propose approaches to obtain more efficient clustering. Most algorithms construct a virtual backbone based on the connected dominating set concept (CDS) [29]. From these algorithms we can talk about PACDS or Power Aware Connected Dominating Set [30], ECDS Energy Efficient Distributed Connecting Dominating Sets [31] and TMPO or Topology Management by Priority Ordering [32].

\section{E.4. Hybrid approaches}

The hybrid approach for topology control uses in general a combination between a clustering method and other techniques such as CLUSTERPOW algorithm [33] which is an improvement of COMPOW mentioned above. This algorithm defines several levels of clusters with different radio powers for communication within and between clusters as shown in Fig.4.

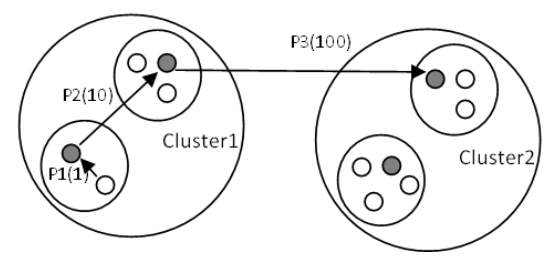

Fig.4. Principle of the CLUSTERPOW algorithm

A recent hybrid method of topology control using power adjustment and hierarchical structure of a wireless 
network is proposed in [34] by S. T. Afshord and al. (2013) which is a cluster-based topology control algorithm called CLNeigh as combination of graph theory concepts and neighbor-based topology control. It builds an energy-efficient and a low interference topology.

\section{E.5. CTP protocol}

As cited in the beginning of this paper, we will compare the performances of our approach with the Collect Tree Protocol CTP [35].

CTP is a simple tree based protocol proposed by O.Gnawali and al. [36] and implemented in TinyOS. CTP is a routing protocol that computes any cast routes to a single or a small number of designated sinks in a wireless sensor network basing only on a network parameter which is the link quality estimation. It uses periodic messages called beacons to maintain topology. A beacon is a packet that contains the link quality estimation between tow nodes. For topology change, a node uses this value to choose its parent from candidate neighbors. Topology control in CTP consists to change parents of nodes (next hop to the sink) when it is necessary. Each node maintains in its routing table a metric called ETX (expected transmissions) as its routing gradient. The ETX of a node is the ETX of its parent plus the ETX of its link to this parent. It is used for validate a route by selecting the parent which have the lowest ETX value. The routing frame format is shown in Fig.5:

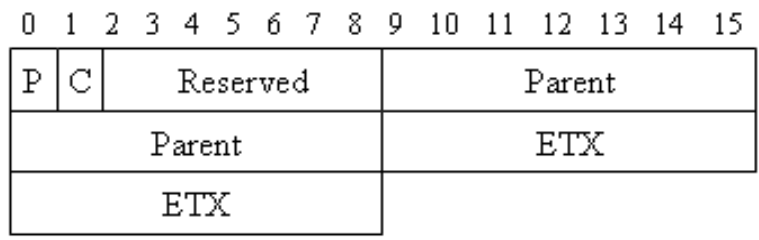

Fig.5. CTP routing frame

Where $\mathrm{P}$ is a bit used to request routing information from other nodes, $\mathrm{C}$ is a bit used for congestion notification, Parent is the node's current parent and ETX is the node's current routing metric value. The software architecture of CTP contains three subcomponents (Fig.6):

LE for link estimator, which is responsible for estimating the single-hop ETX of communication with single-hop neighbors.

$\mathrm{RE}$ for routing engine, which uses link estimates as well as network-level information to decide which neighbor is the next routing hop.

FE for forwarding engine, which maintains a queue of packets to send. It decides when to send them. The forwarding engine is responsible for forwarded traffic as well as traffic generated on the node.

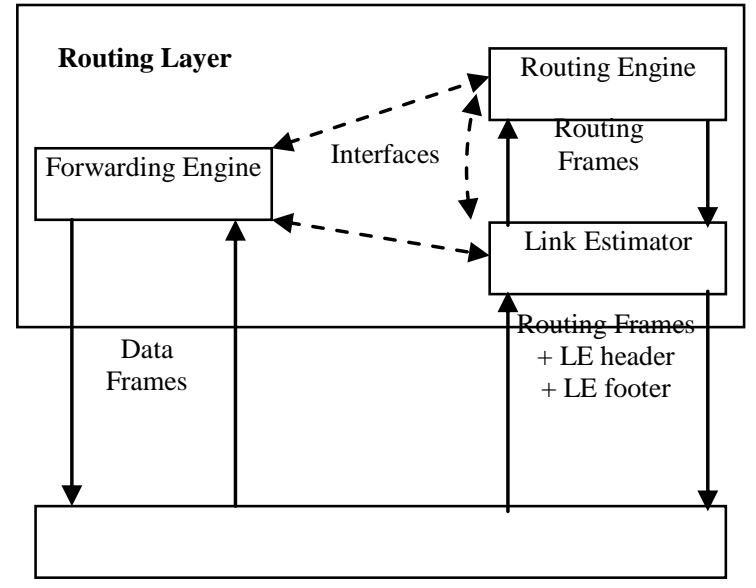

Fig.6. CTP components interaction

The estimation of ETX values for one hop is task of link estimator. For each neighbor, LE determines this value by considering link quality of incoming and outgoing links as follows:

The quality of outgoing links QS between node $\mathrm{v}$ and its neighbor $\mathrm{Ni}$ is done by:

$$
Q S v(N i)=\operatorname{Pout}(v) / \operatorname{Poutc}(v)
$$

Similarly, the quality of incoming links QE between a node $v$ and its neighbor $N_{i}$ :

$$
Q E v\left(N_{i}\right)=\operatorname{Pin}(v) / \operatorname{Pout}(v)
$$

The node $v$ calculates the ETX value in function of quality $Q$ of an outgoing or incoming link (QS or QE) as follows:

$$
\operatorname{ETX}(v)=\left(\alpha_{E T X} \cdot Q+\left(1-\alpha_{E T X} \cdot Q\right) \cdot E T X_{\text {old }}\right)
$$

Where $\alpha_{T D}$ is a weighting constant. Finally, the equations for calculating $E T X$ are:

$$
\begin{gathered}
E T X_{\text {root }}=\text { O for a sink } \\
E_{T X_{\text {node }}}=E_{T} X_{\text {parent }}+E_{\text {tinktoparent }}
\end{gathered}
$$

CTP should choose the route with the lowest ETX.

\section{PROPOSED METHOD}

\section{A. The problem}

Our contribution consists to propose a hybrid and distributed method using MAS for wireless sensor 
network topology control based on local decisions taken by the node itself, function of several parameters: residual energy, number of neighbors, links quality, etc. The main objective is to have at any time a connected, homogeneous and fault-tolerant network which should be capable to predict and avoid as much failures as possible. This method is inspired from influence systems, and requires strong cooperation between nodes.

When transmitting data, the principle consists to select the most fault tolerant nodes that ensure safe transfer; we shall see that the resulting network gets a long duration connected network property. The use of MAS seems to be a suitable approach according to the distributed, cooperative and emergent principle that characterizes this operation.

The role of the used MAS is to calculate for each node a parameter determining its state and its capacity to continue to go further without risk of failure such as energy depletion or congestion, this have to be done before the end of data transfer. The defined calculated parameter is said the tolerance degree of a node.

Some works in this context have a similar principle which is based on computing one or more metrics to control the topology such as [37] that calculates the probability of node's failure, [38] which uses an algorithm to predict the lifetime of each node and also [35] based on the evaluation of the link quality between two nodes. So the main difference between these works is the choice of network parameters and how to calculate the value in question. In our approach, we propose another method where we will use the link quality evaluation of [35] then we add the battery status and the number of hops to the Sink which is also considered while a topology change. This principle allows us to express at the same time the life time and the fault tolerance by using the battery state, the link quality, and the choice of best path by integration of the measurement of the number of hops. A thresholding mechanism is implemented to avoid frequent changes in the topological due to minor differences.

Before detailing our proposal, we mention recent works in the context of topologies with fault tolerance. We note that these works do not use agent technology.

\section{B. Related works}

We are interested here in the works on the topology control which are based on local settings to predict or estimate other values or states or to take decision in order to optimize performances.

We start with [37] of Yin Rong-rong and al. (2012) which proposed an adaptive method for topology control with fault tolerance by calculating the node failure probability $F P$ based on the ratio of the consumed energy $E_{c}$ and the initial energy $E_{\text {init }}$ and another fault probability $P$ associated to hardware and software components, and we have :

$$
F P=P . E_{c} / E_{\text {init }}
$$

In [39], Dario Bruneo and al. (2012) shows that the introduction of Markov techniques allows to estimate the lifetime taking a node active-sleep cycle as a model of transitions with probabilities for each transition. This allows predicting defects. But in reality, lifetime also depends on the node activities when it is at active state.

Another work using Markov models [40] of Arslan Munir and al. (2011) discussed Markov modeling for fault tolerant wireless sensor networks where the node's degree of tolerance is estimated according to the number of deployed nodes and the probability of replacement of all in case of failure.

Failures in a sensor network can be detected by application of "fuzzy inference" according to [41] of Safdar Abbas Khan and al. (2012) where the sensor measures are compared with expected values by a neural network. The differences in behavior allow to detect anomalies.

The lifetime of a hierarchical network is studied by BoChao Cheng and al. (2012) in [38]. It proposes an algorithm for lifetime prediction in better and worst cases in a WSN with one hop clusters where the cluster heads should communicate directly with the sink. The objective of this work is to find the best deployment for this topology type.

A cooperative approach for topology control is proposed by Paolo Costa and al. (2009) in [42]. The construction of the topology consists to choose nodes that guarantee a degree of k-connectivity by using a minimum radio range.

The stability of topology is obtained by cooperation between nodes to consent on the radio level under the constraint of k-connectivity.

\section{Proposal details}

\section{C.1.The network model}

Related to an application domain, the nodes of network are deployed randomly in a known field to capture specific types of information; the captured data are then transmitted to a control station or sink. When transferring data, the choice of path is based on the choice of the next hop or parent from the current node obeying to some routing protocol. Here, the next hop is selected from the neighbors using the degree of tolerance at the time of transfer, so the node that has the highest degree of tolerance will be qualified for this transfer. We will detail later how to calculate this degree of tolerance. This task is performed by agents implanted on nodes. Fig.7 shows this principle where the agent is responsible for selecting the parent of the node that wants to transmit packets from neighboring candidates. Some nodes may become critical, so they can't be parents of other nodes. 


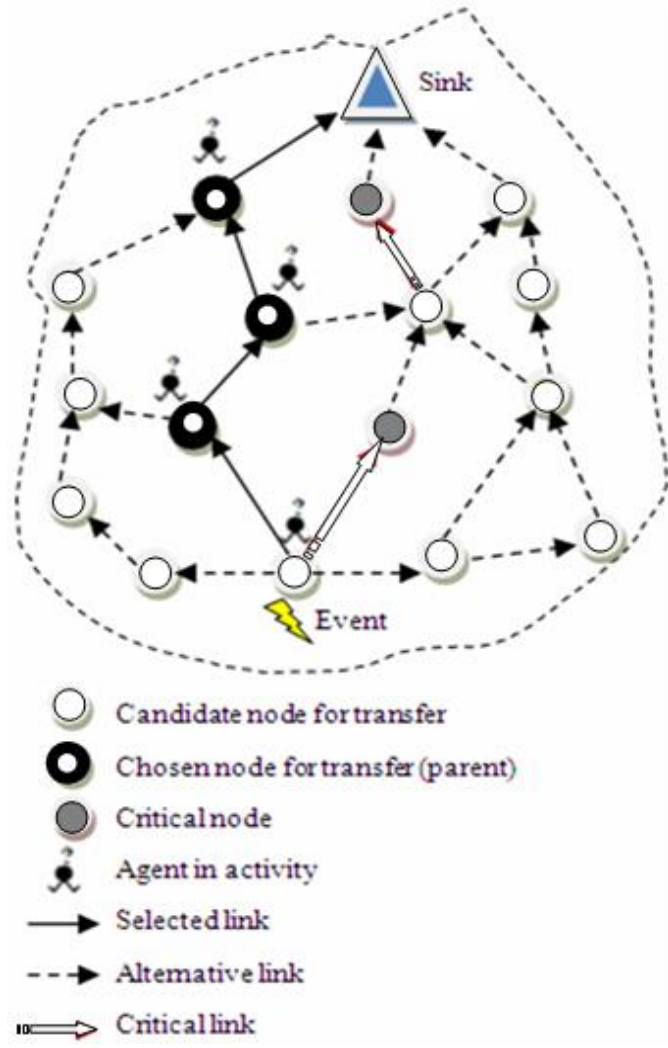

Fig.7. Network model

\section{C.2. Topology construction}

After deployment of nodes, the sink diffuses an initialization message Init based on the hop count value $\mathrm{HC}$ which is zero for the sink. The neighborhood discovery is included in this method. Each node $n$ which receives the Init message considers the sender of the message as the next hop for the next transmissions if the $\mathrm{HC}$ value of this last is less than the receiver node n. So, it does:

\section{if $H C(n)>H C$ (init) then $H C(n) \leftarrow H C$ (init)}

Then it rebroadcasts the Init message. At the beginning, the $\mathrm{HC}$ values are set to infinity for all nodes except the sink which is initialized to zero. This principle allows the construction of a tree for transmitting data to the sink.

\section{C.3. Topology control method}

After the stability of the topology obtained by initialization process (there is no node which rebroadcasts the Init message), nodes calculate their degree of tolerance as follows, we denote:

- $\mathrm{E}_{\text {init }}$ : initial energy of a node;

- $\mathrm{E}_{\mathrm{v}}$ : residual energy of the node $\mathrm{v}$;

- $N_{v}$ : set of neighbors of $v, N_{i} \in N_{v}$ is a neighbor of $v$;

- Pin $\left(\mathrm{N}_{\mathrm{i}}\right)$ : number of received packets by $\mathrm{v}$ from $\mathrm{N}_{\mathrm{i}}$ during a period $\mathrm{t}$;
- Pout $\left(\mathrm{N}_{\mathrm{i}}\right)$ : number of broadcast packets by $\mathrm{N}_{\mathrm{i}}$ during a period $\mathrm{t}$;

- Poutc $\left(\mathrm{N}_{\mathrm{i}}\right)$ : number of correctly received packets by the neighbors of $\mathrm{Ni}$ (with acknowledgment);

- HC(v) : hop count from $v$ to the sink;

- $\mathrm{NH}(\mathrm{v})$ : the next hop from $\mathrm{v}$ to the sink also said parent of $\mathrm{v}$;

For the calculation of tolerance degree TD, we propose to use the link quality and battery status of each node.

The calculation of the first parameter is inspired from the link quality estimation used in [35] and defined by formulas (1), (2) and (3) of section II.5.5 of this paper. For battery status we consider the relationship between the residual energy and the initial one.

The node $\mathrm{v}$ calculates its tolerance degree $\mathrm{TD}$ in function of quality $\mathrm{Q}$ of an outgoing or incoming link (QS or QE) and the battery status of the node in question as follows:

$$
T D(v)=\left(E_{v} / E_{\text {init }}\right) \cdot\left(\alpha_{T D} \cdot Q+\left(1-\alpha_{T D} \cdot Q\right) \cdot T D_{\text {old }}\right)
$$

Where $\alpha_{\mathrm{TD}}$ is a weighting constant that can take values between 0 and 1 ( 0.9 in our case).

The parent change procedure is based on the parameter values of the old parent and candidate neighbors. We use mainly the energy $\mathrm{E}$, the degree of tolerance TD (old value) and the hops count HC. To avoid parent change when small variations in these parameters happen, a thresholding principle is used for each one. So the node $\mathrm{v}$ decides to choose a neighbor $\mathrm{Ni}$ as its new parent $\mathrm{NH}$ if the following conditions are satisfied:

$$
\begin{array}{ll}
- & \mathrm{HC}(\mathrm{Ni}) \leq \mathrm{HC}(\mathrm{v})+\text { Threshold_HC } \\
\text { - } & \mathrm{E}(\mathrm{Ni})>\mathrm{E}(\mathrm{NH}(\mathrm{v}))+\text { Threshold_E } \\
\text { - } & \mathrm{TD}(\mathrm{Ni})>\mathrm{TD}(\mathrm{NH}(\mathrm{v}))+\text { Threshold_TD }
\end{array}
$$

It is necessary to know that the verification order of these conditions is very important; it also depends on the nature of application using our approach. Here, we chose the hops count in first with a threshold that depends on the network size (number of nodes in the network) in order to avoid having long paths and therefore delaying the traffic. This may be not useful if the application has no time constraint.

The algorithm shows the parent selection process according to the previous conditions. $\mathrm{T}_{\mathrm{E}}, \mathrm{T}_{\mathrm{TD}}$ and $\mathrm{T}_{\mathrm{HC}}$ represent the threshold values for the energy, degree of tolerance and the hops count respectively. The function Change_Parent represents a switching tool between the active parent and the candidate one, the TD function implements the degree of tolerance estimation. In this algorithm, the use of cited parameters is presented as a control system which able to configure how the protocol works according to the goals and objectives of the WSN application. 


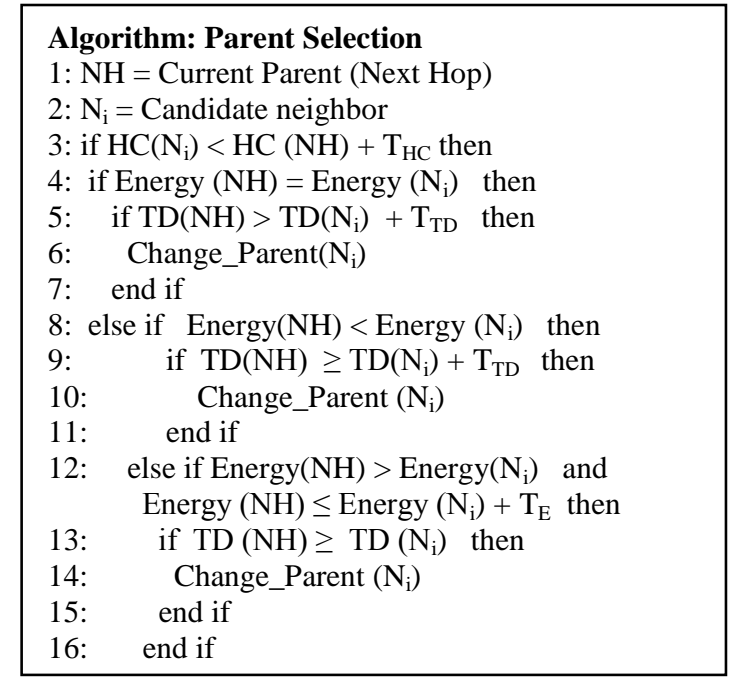

\section{IMPLEMENTATION AND RESULTS}

\section{A. Simulation}

In order to validate the proposed solution in this paper and study its performances as well as the adaptation of a multi-agent model for this type of distributed algorithm, we have implemented our approach using the Castalia simulator [43] which is based on the simulator Omnet ++ [44]. Castalia is a commonly used tool in recent years for WSNs simulation due to its gratuity and easy integration of new protocols in its software layer structure and also its configurable environment as needed. For our study, we used version 3.2 of Castalia with Omnet ++4.2 with an ubuntu Linux machine. Our distributed algorithm is implemented as an agent implanted on each sensor node. The sensors form MAS where cooperation is provided by exchanging messages at the time of data transfer to select the most fault tolerant nodes and that at each hop. It is clear that the principle of our algorithm requires that agents must communicate the necessary information like the latest values of tolerance degree, energy and the number of hops. The diagram in Fig. 8 below shows that the agent on node 3 for example has the choice to transfer its packets through nodes 2,6 or 7 depending on the status of each one of these nodes.

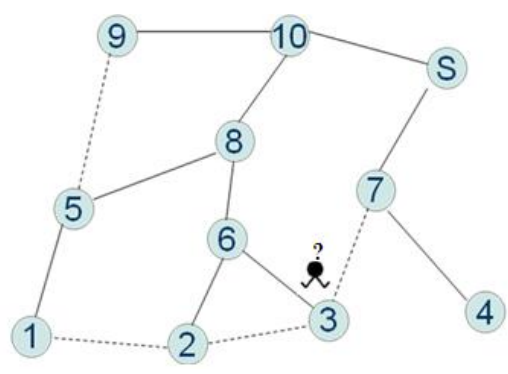

Fig.8. Principle of our algorithm

\section{B. Hypothesis}

To perform simulations, we consider the following hypothesis:
- Initially, each node has an initial energy.

- All sensor nodes are battery powered with limited energy, except the Sink.

- Network size is specified at the beginning of each simulation, node 0 is chosen as Sink.

The table 1 below shows other simulation parameters:

Table 1. Wsn used parameters

\begin{tabular}{|l|l|}
\hline \multicolumn{1}{|c|}{ Parameter } & \multicolumn{1}{|c|}{ Value } \\
\hline Number of nodes & Up to 300 \\
\hline Field Deployment & $250 \times 250$ meters \\
\hline Deployment type & Random \\
\hline Radio model & CC2420 \\
\hline Radio power & $0 \mathrm{dBm}$ \\
\hline Initial energy & 18720 joules \\
\hline Simulation time & $100,200,300, .1000 \mathrm{sec} .$. \\
\hline Thresholds: $\mathrm{T}_{\mathrm{HC}}, \mathrm{T}_{\mathrm{E}}$ and $\mathrm{T}_{\mathrm{TD}}$ & $20 \%$ of difference for each \\
\hline
\end{tabular}

\section{Results and analysis}

To demonstrate the performance of our approach which uses a WSN based on multi-agent system with fault tolerance and quality of service, we make a comparison with CTP protocol [35]. On figures, our approach is noted ATC for agent topology control.

Fig.9 illustrates the execution of our protocol and shows that traffic is distributed across all nodes. This is assured by changing the parents according to the parameters of each node. For example:

- (A): Construction of the initial tree

- (B): Node 7 becomes a parent of 3

- (C): Node 3 becomes a parent of 2

- (D): Node 2 becomes a parent of 3 and 1 becomes a parent of 2

- (E): Node 9 becomes a parent of 5

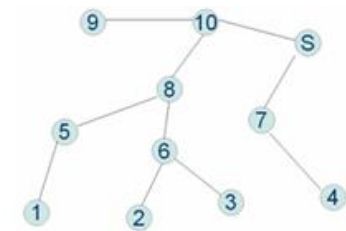

(A)

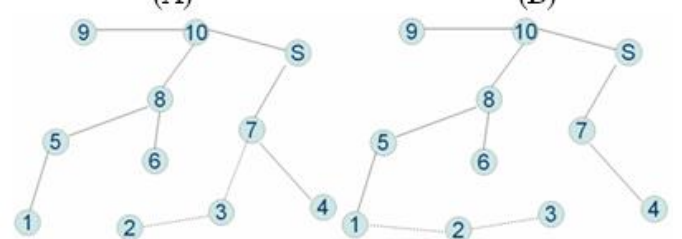

(B)

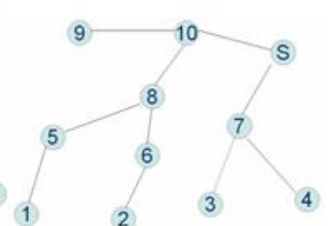

(B)

(D)

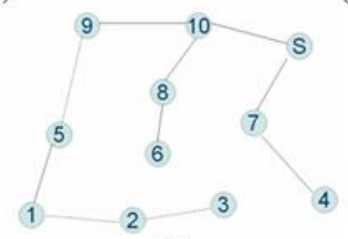

(E)

Fig.9. Illustration 


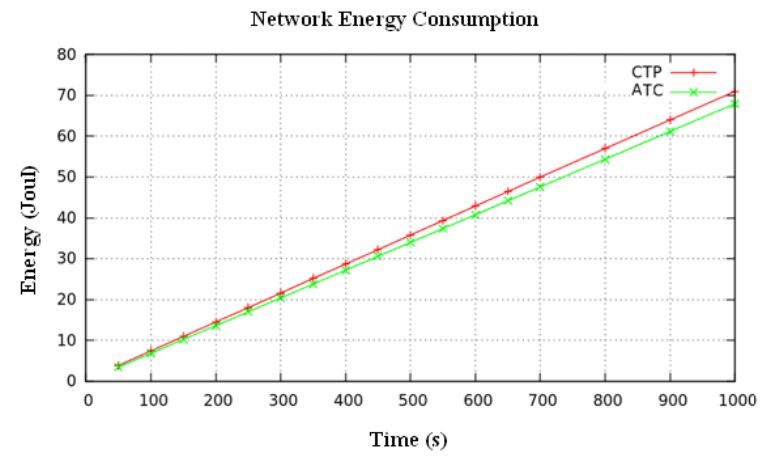

Fig.10. Impact on energy consumption

Fig.10 shows a small deference in energy consumption using CTP and ATC. This deference is notable in networks with big size. Indeed, our solution uses a mechanism similar to CTP but using an other formula to calculate the degree of tolerance which allows to select parents with an other manner. So, the network consumes its energy according to position and states of each node and its neighbors.

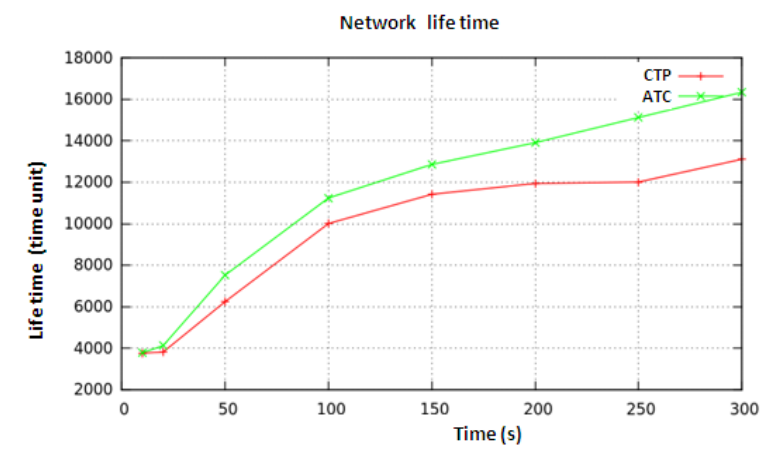

Fig.11. Impact on life time

The curves in Fig. 11 show a notable divergence in case of WSN with big size, indeed, the high density of nodes allows our approach to find other paths to conserve energy of those used. However in CTP, congestion or over-use of a path leads to the premature death of nodes.

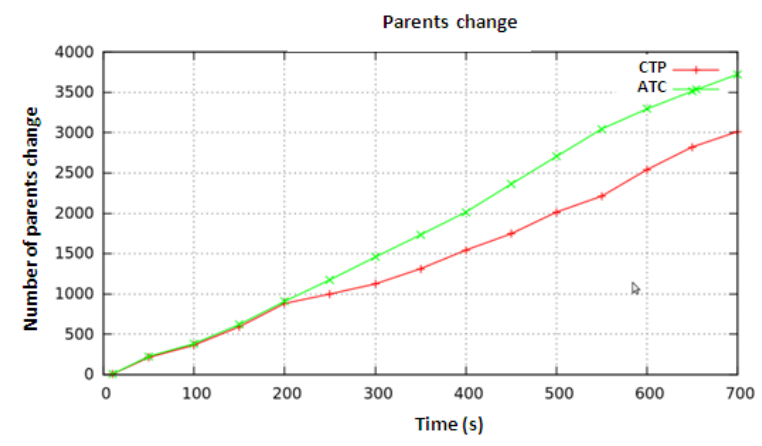

Fig.12. Parent change in the time

We note from Fig.12 that the difference in the number of parents change in both cases is not important at first, and over time a divergence become more important, it is explained by the diminution of degree of tolerance calculated by our protocol, which requires changes in parents in order to ensure a loads balancing.

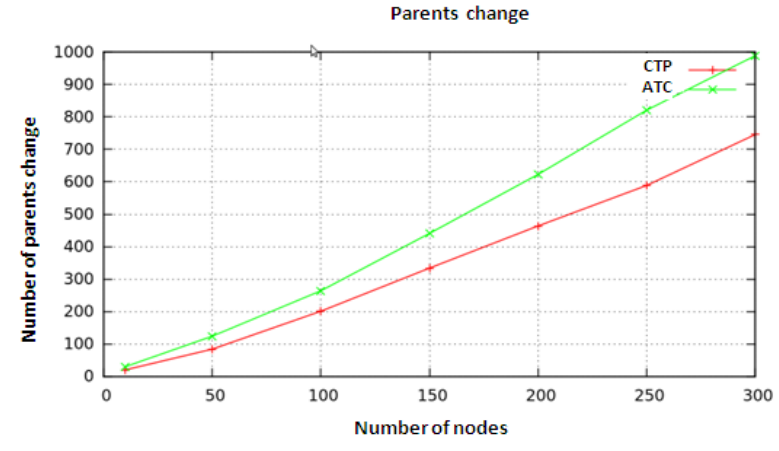

Fig.13. Change of parents with network size

Fig.13 also shows the curves of parent change, but this time based on the network size. By increasing the size, we observe a difference in the number of parent changes relative to the two protocols. It is higher for large-scale networks that ensure the existence of other paths where the best one will be selected. The use of several parameters by our protocol gives more opportunities than CTP which use only the quality of links.

\section{CONCLUSION AND PERSPECTIVES}

In this paper, we presented a state of the art on the use of MAS in wireless sensor networks. According to the status of node and its neighbors, the main objective is to have a fault-tolerant network and extend its lifetime by optimizing the choice of paths based on changing parent method. The new concept of degree of tolerance has been used in the choosing parent and/or neighbor procedure. To respect multi-agent spirit, this is achieved in a cooperative and distributed manner. Therefore, a multiagent system seems more adapted. The implementation using Omnet++/Castalia of our proposal shows the relevance of multi agent systems approach compared with classical method like CTP.

Indeed, the simulation results show that our solution leads to assess at any time the level of fault tolerance of each node which improves the paths selection process and therefore the life time of nodes. The performance of our approach can be enhanced by providing a formula that uses other network parameters to calculate the degree of tolerance or even use a probabilistic approach to predict the activities of a sensor node such as packet traffic. Other perspective works could concern to embed security parameters and develop more complex formulas. The test of our protocol on a real platform is also the aim of further applications.

\section{REFERENCES}

[1] S.Hussain, E.Shakshuki, and A.W.Morning, "Agent-based system architecture for wireless sensor networks", In Proceedings of 20th International Conference on Advanced Information Networking and Applications, Vienna University of Technology, Vienna, Austria, 18-20 April 2006.

[2] J.P.Jamont, and O.Michel, "Design of embedded multi agent systems: discussion about some specificities", VII Agent-Oriented Software Engineering Technical Forum, 
Paris, French, December 15, 2010.

[3] M.Samir," Further Structural Intelligence for Sensors Cluster Technology in Manufacturing", Journal of Sensors, Vol.6, 2006, pp.557-577.

[4] K.B. Pratik, Q.Hairong, and Y. Xu, "Collaborative Mobile-agent-based sensor fusion", Information Fusion, Vol.9, 2008, pp.399-411.

[5] J.P.Jamont, "MWAC: Multi-Wireless-Agent Communication", Revue Technique et science informatiques RSTI série TSI, Vol. 25, N5, 2006, pp. 661-688.

[6] K.Smarsly, K.H.Law, and M.König, "Resource-Efficient Wireless Monitoring based on Mobile Agent Migration", In proceedings of the SPIE: Health Monitoring of Structural and Biological Systems 2011. San Diego, CA, USA, 06 March 2011.

[7] R.H.B. Rahal, and B.Bounabat, "Wireless Sensor Network Simulation Of The Energy Consumption By A Multi Agent System", Journal of Theorical and Applied Information Technology, Vol. 25, N 1, 2011.

[8] W.Xue, B.Dao, D.Liang, and W.Sheng, "Collaborative Target Classification and Localization in Wireless Sensor Networks Agent". Journal of Sensors, Vol.7, N 8, 2007, pp.1359-1386.

[9] Y.Lingyun, W.Xingchao, G.Jianhou, and Z.Yanfang, "Data Gathering Algorithm Based on Mobile Agent and Emergent Event Driven in Cluster-Based WSN", Journal of Networks, Vol.5, N 10, 2010, pp.1160-1168.

[10] N.Jabeur, and P. A.Graniero, "Agent-Based Clusters to Virtually Manage Spatially Distributed Sensors", Projects TDMDSD08 and SII-73 University of Windsor, Department of Earth and Environmental Sciences, Windsor, Ontario, Canada, 2008.

[11] L.Min, X.Shijun, and S.Siyi, "An agent-based routing algorithm assistedQoS-forwireless sensor networks", Journal of Network and Computer Applications, Vol.35, N 1, 2012, pp.29-36.

[12] B.Dario, S.Marco, B.Andrea, C.Davide, and G.Marco, "Markovian agent modeling swarm intelligence algorithms in wireless sensor networks". Journal of Performance Evaluation, Vol.69, N 3-4, 2012, pp.135-149.

[13] D.C.Matthew, A.Djamel, P.H.Barry, and H.Alan, "A Bayesian network approach to a biologically inspired motion strategy for mobile wireless sensor networks", Journal of Ad Hoc Networks, Vol.7, N 6, 2009, pp.12171228.

[14] V.Saamaja, K.R.Kiran, and R.M.C.Siva, "Using mobile data collectors to Improve network lifetime of wireless sensor networks with reliability constraints", Journal of Parallel and Distributed Computing, Vol.70, N 7, 2010, pp.767-778.

[15] G.Wenzhong, X.Naixue, C.Han-Chieh, H.Sajid, and C.Guolong, "Design and Analysis of Self-Adapted Task Scheduling Strategies in Wireless Sensor Networks". Journal of Sensors, Vol.11, 2011, pp.6533-6554. doi: $10.3390 / \mathrm{s} 110706533$.

[16] X.Wang, B.Dao-wei, D.Liang, and W.Sheng, "Multiagent Negotiation Mechanisms for Statistical Target Classification in Wireless Multimedia Sensor Networks", Journal of Sensors, Vol.7, N 10, 2007, pp.2201-2237; doi: $10.3390 / \mathrm{s} 7102201$.

[17] H.Hai-ping, W.Ru-chuan, S.Li-juan, W.Hai-yuan, and X.Fu, "Research on tasks schedule and data transmission of video sensor networks based on intelligent agents and intelligent algorithms", The Journal of China Universities of Posts and Telecommunications, Vol. 16, N 6, 2009, pp.84-91.
[18] F.Giancarlo, G.Stefano, G.Raffaele, and G.Antonio, "Agent-based Development of Wireless Sensor Network Applications", In Proceedings of the 12th Workshop on Objects and Agent, (741), Rende (CS), Italy, Jul 4-6, 2011,pp. 123-132.

[19] B.Rijubrata, "Mobile Agent based Architecture for. Wireless Sensor Networks", In proceedings of TKK T110.5190 Seminar on Internetworking, Helsinki University of Technology, Finland, April 27, 2009.

[20] K.B.Ratik, S.Mendel, and P.Shashi, "An agent-oriented information processing architecture for sensor network applications", International Journal of Ad Hoc and Ubiquitous Computing, Vol.1, N 3, 2006, pp.110-125.

[21] A. M. Popescu, I. G. Tudorache, B. Peng and A.H. Kemp, "Surveying Position Based Routing Protocols for Wireless Sensor and Ad-hoc Networks", International Journal of Computer Network and Information Security (IJCNIS), Vol.4, N 1, April 2012, pp.41-67.

[22] A.A.Azrina, Y.A.Sekercioglu, P.Fitzpatrick, and M.Ivanovich, "A survey on distributed topology control techniques for extending the lifetime of battery powered wireless sensor networks", Communications Surveys and Tutorials, IEEE, Vol.15, N 1, 2013, pp.121-144, on line: http://dx.doi.org/10.1109/SURV.2012.031612.00124.

[23] V.Rodoplu, and T.H.Meng, "Minimum energy mobile wireless networks", IEEE J. Salt. Areas Communications, Vol.17, N 8, 1999, pp.1333-1344.

[24] L.Li, and J.Y.Halpern, "Minimum-energy mobile wireless networks revisited". In proceedings of IEEE International Conference on Communications, Helsinki, Finlan, 11-14 Jun 2001, pp. 278-283.

[25] S.Narayanaswamy, V.Kawadia, R.S.Sreenivas, and P.R.Kumar, "Power control in ad-hoc networks: Theory, architecture, algorithm and implementation of the protocol compow", In proceedings of European Wireless Conference (2002), pp.156-162.

[26] X.Ya, J.Heidemann, and D.Estrin, "Geography-informed energy conservation for ad hoc routing", In proceedings of 7th Annual international conference on mobile computing and networking-MOBICOM, New York, USA, 2001, pp.70-84.

[27] C.Schurgers, V.Tsiatsis, and M.B.Srivastava, "STEMtopology management for energy efficient sensor networks", In proceedings of 2002 IEEE Aerospace Conference Proceedings, Vol.3, pp.3-1099-3-1108, On line: http://dx.doi.org/10.1109/AERO.2002.1035239.

[28] A.Cerpa, and D.Estrin, "Ascent: Adaptive SelfConfiguring sensor networks topologies", IEEE Transactions on Mobile Computing, Vol.3, N 3, 2004, pp.272-285.

[29] D.Z.Du, and P.Pardalos, "Handbook of Combinatorial Optimization", Kluwer Academic Publishers, 2004.

[30] M.Burkhart, R.Wattenhofer, and A.Zollinger, "Does topology control REDUCE interference? ", In proceedings of. 5th ACM International Symposium on Mobile Ad Hoc Networking and Computing, New York, NY, USA, ACM 2004,pp.9-19.

[31] Z.Yuanyuan, X.Jia, and H.Yanxiang, "Energy efficient distributed connected dominating sets in wireless sensor networks building", In proceedings of the 2006 International conference on wireless communications and mobile computing, New York, NY, USA, ACM 2006, pp.797-802.

[32] L.Bao, and J.J.Garcia-Luna-Aceves, "Topology management in ad hoc networks", In proceedings of. 4th ACM International Symposium on Mobile AdHoc 2003, Networking and Computing, ACM New York, NY, USA, 
pp. 129-140.

[33] V.Kawadia, and P.R.Kumar, "Power control and clustering in ad hoc networks", In INFOCOM 2003. Twenty-second annual joint conference of the IEEE Computer and Communications. IEEE Societies, 30 March-3 April 2003, pp. 459-469.

[34] S. T. Afshord, B. Zarei, and B. Arasteh, "A New Distributed and Power-Efficient Topology Control Algorithm for Wireless Ad-Hoc Networks", International Journal of Computer Network and Information Security (IJCNIS), Vol.12, 2013, pp.20-26.

[35] G.Omprakash, F.Rodrigo, J.Kyle, M.David, and L.Philip, "Collection Tree Protocol", In proceedings of the 7th ACM Conference on embedded networked sensor systems, Berkeley, CA, USA, November 2009, pp.1-14.

[36] G.Omprakash web page on CTP protocol, Consulted Janaury 27, 2014, from http://sing.stanford.edu/gnawali/ctp/.

[37] Y.Rong-rong, L.Bin, L.Ya-qian, and H.Xiao-chen, "Adaptively fault-tolerant topology control algorithm for wireless sensor networks", The Journal of China Universities of Posts and Telecommunications, Vol.19, $\mathrm{N}^{\circ}$ Suppl. 2, 2012, pp.13-18.

[38] C.Bo-Chao, L.Guo-Tan, T.Ryh-Yuh, and H.Ping Hsu, "Network lifetime bounds for hierarchical wireless sensor networks in the presence of energy constraints", Journal of Computer Networks, Vol.56, N 2, 2012, pp.820-831.

[39] B.Dario, D.Salvatore, L.Francesco, P.Antonio, and S.Marco, "Evaluating wireless sensor node longevity through Markovian techniques", Journal of Computer Networks, Vol.56, N 2, 2012, pp.521-532.

[40] A.Munir, and A.Gordon-Ross, "Markov Modeling of Fault-Tolerant Wireless Sensor Networks", In Proceedings of 20th International Conference on Computer Communications and Networks, Maui, HI, July 31 2011-Aug. 4 2011, pp. 1-6.

[41] A.K.Safdar, D.Boubaker, and D.Karim, "Application of fuzzy inference systems to detection of faults in wireless sensor networks", Journal of Neurocomputing, Vol.94, N 1, 2012, pp.111-120.

[42] C.Paolo, C.Matteo, B.Stefano, and C.Luca, "A Cooperative Approach for Topology Control in Wireless
Sensor Networks", Pervasive and Mobile Computing, Vol.5, N 5, 2009, pp.526-541.

[43] A.Boulis, "Castalia, A Simulator for Wireless Sensor Networks and Body Area Networks", Version 3.2. User's Manual, NICTA March 2011.

[44] OMNeT++ Homepage. http://www.omnetpp.org.

\section{Authors' Profiles}

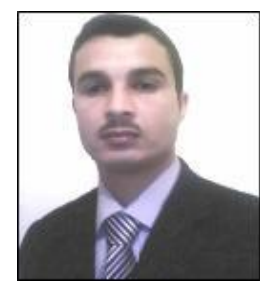

Bechar Rachid obtained his engineer degree in computer science from the University of Mostaganem, Algeria in 2003 and the degree of Magister in computer science from the same University in 2010. He worked at the University of Mostaganem as an engineer in software development from 2004 to 2010. In 2011 he joined the University of Chlef, Algeria as a maitre assistant in the computer science department where he is working now. He is a PhD student at the University of Oran Essenia, Algeria. His research interests include computer networks, wireless sensor networks and image processing.

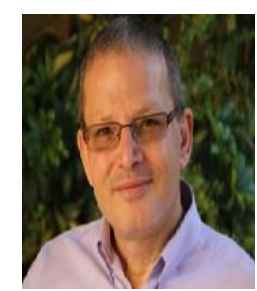

Haffaf Hafid obtained Doctor degree in computer Science in 2000; is a senior lecturer at the University of Oran Es-Senia (Algeria). He actually heads the R.I.I.R Laboratory at Computer science department - Oran University-. His researches concern different domain as Automatic control and diagnosis, optimisation reconfiguration using matroid theory, system approaches and their applications in Bond graph and monitoring. He has many collaborations projects with European laboratory: Polytech lille where he worked in Intelligent transport systems infrastructures- and LIAUPau (France) in the domain of Wireless sensor Networks (CMEP project).

How to cite this paper: Bechar Rachid, Haffaf Hafid,"Distributed Monitoring for Wireless Sensor Networks: a MultiAgent Approach", IJCNIS, vol.6, no.10, pp.13-23, 2014. DOI: 10.5815/ijcnis.2014.10.02 